\title{
MACROSCOPIC EFFECTS OF SURFACE ROUGHNESS IN CONFINED AIR-FLOW
}

\author{
by \\ P. Bourgin and M. Boutaous \\ Ecole Supérieure de Plasturgie \\ FRANCE
}

\begin{abstract}
One challenge when processing flexible media such as plastic films is to obtain rolls without any aspect defect : if one considers that a "defect" (i.e. wrinkling or buckling) is due to the fact that the stress generated within the roll is greater than some "plasticity yield", then it is crucial to predict the internal stress state.

Several process parameters must be carefully mastered (winding tension, velocity, etc.) as well as the material pertinent properties. One key issue is to optimize the surface topography of the flexible medium so that to improve the quality of the wound roll.

We propose here new parameters which describe the surface roughness of plastic films fairly well. The measurements were carried out by using a $3 \mathrm{D}$ roughness measurement device.

A mathematical model based on homogenization techniques is proposed, where the heights of the roughness peaks, their diameter and their spatial distribution are the governing parameters.

Sampling at different levels is carried out by expressing the percentage of peaks which exceed some given threshold value.

For each tested film, the threshold value will be the only adjustable parameter.

Introducing these parameters into the mathematical model which predicts the evolution of the squeezed air layer and comparing to the experimental data, the following results are obtained :

- It is possible to adjust one single parameter so that to obtain a very good agreement between the experimental data and the theoretical results.

- The smoother the film, the more important the highest peaks are in terms of air leakage.
\end{abstract}




\section{I - INTRODUCTION}

The overall quality of wound materials (i.e. plastic, paper, steel ...) is strongly connected to the roll quality which in turn depends on the residual stresses within the roll [1]. In order to take into account the air entrapped between the web layers, several authors modeled the roll like an orthotropic material with a non-linear radial elastic modulus. In addition, it is well established that the residual air layers depend on the surface roughness.

In a previous work [2], the influence of the surface roughness on the air flow kinetics was studied by squeezing a rough plastic film on a smooth rigid plate. In order to quantify this influence, a mathematical model, based on homogenization techniques, was developed. In this model, we represent the surface roughness like a periodic array of cylinders. The macroscopic experimental data can be nicely fitted by adjusting some microscopic parameters such as the cylinder diameters, or their heights.

The question which arises now is : how to connect these parameters to the real configuration of the surface roughness of the films?

To provide a first answer, we have tested two types of PET films, and found an interesting correlation between the micro and macro experiments.

\section{II - PRINCIPLE OF ROUGHNESS MEASUREMENT}

The profilometer used is equipped with a stylus moving on the surface to be tested. The vertical displacement of the apex of the stylus (whose radius of curvature is of the order of one tenth of a micrometer) is analyzed numerically to determine the heights of the bumps. Square PET film samples $(3 \mathrm{~mm} \times 3 \mathrm{~mm})$ are tested. Continuous measurements are taken over a width, and are repeated every $10 \mu \mathrm{m}$ in order to scan the whole surface.

The 2D profile obtained at each traveling is numerically treated so that to remove the defects due to the wavy surface of the film. Mineral fillers are generally incorporated into the polymer during processing so that to generate the film surface roughness. These fillers form agglomerates (Figure 1). The statistical data processing which is carried out basically consists in selecting the peaks higher than some prescribed threshold. The number of peaks which are taken into account obviously decrease as the threshold level increases. It is then possible to determine the percentage of peaks higher than the threshold value (from which it is deduced the mean distance between the peaks) as well as the mean values of their heights and widths.

These parameters are introduced into the mathematical model which is based on the representation of the surface roughness by means of a regular array of cylinders. They represent respectively : cylinders heights, spacing between cylinders and diameters. 


\section{III - SQUEEZING SET-UP}

Only the basic features of the experimental set-up are summarized here, a more detailed description being found in previously published work $[3,4]$.

A polished glass disk is put on a flat support having a circular slit connected to a vacuum pump : see Figure 2.

A sample of plastic film (i.e. PET) is displayed on the glass plate and subambient pressure is applied by operating the vacuum pump. The air layer which initially separates the film from the glass plate is partially evacuated : a quasi circular front starts from the slit and propagates towards the center as seen in figure 3. The reduction of the air interlayer is easily determined by using elementary optics laws.

It was observed that for any sample and any set of operating conditions the instantaneous thickness of the air layer $e(t)$ at the center of the front is of the form $: e(t)=e_{i}-k$. $t$, where $e_{i}$ is the initial thickness, $t$, the time and $k$ some parameter characteristic of each sample, that we called "dynamic roughness coefficient".

\section{IV - MATHEMATICAL MODEL}

The objective of the model is to establish a relationship between the thickness reduction of the air layer and the displacement of the front (see figure 4) and then to see how this relationship would depend on the surface topography of the plastic film.

For more details about the model, see [2]. The basic idea consists in computing the front radius evolution versus time by merely applying flow rate conservation between the upstream and the downstream zones separated by the front radius. Note that Reynolds' thin film flow theory is used in the downstream zone, and the flow is considered to be quasi-static, inertialess and the fluid (air) to be incompressible.

Assuming that $e(t)=e_{i}-k . t$ where $k$ is some parameter determined experimentally, function $R(t)$ is governed by the following ordinary differential equation :

$$
k R(t)^{2} \ln \left(\frac{R(t)}{R_{0}}\right)+2 R(t)\left(-e_{i}+k t+e_{f}(t)\right) \ln \left(\frac{R(t)}{R_{0}}\right) \frac{d R(t)}{d t}+\frac{1}{6 \mu} \frac{P a}{\mu} e_{f}^{3}(t)=0
$$

This equation shows a coupling between two unknown time functions: $R(t)$ and $e_{f}(t)$. It is therefore necessary to introduce an additional equation. For that purpose, the equation which governs the confined flow in the zone downstream of the front will be searched for. 
The theory was developed in [2]. The general idea consists in considering a flow through a regular array of identical cylinders confined between two parallel planes and squeezed against each other under a given pressure: see figures 5 and 6. The radii of these cylinders and the distance between their axes are respectively denoted by "a" and " $\varepsilon$ ". The squeeze flow will be studied by using homogeneization techniques in the spirit of Bayada's and Chambat's [5].

The basic equations are:

- Stokes and continuity equations as in [6]

$$
\left\{\begin{array}{l}
\mu \Delta \overrightarrow{\mathrm{u}}=\vec{\nabla} \mathrm{p} \\
\vec{\nabla} \cdot \overrightarrow{\mathrm{u}}=0
\end{array}\right.
$$

where $\mu$ indicates dynamic viscosity

- Adequate boundary conditions:

-no-slip boundary condition,

- prescribed pressure at the edge of the domain,

- local periodicity of velocity.

Writing mass conservation in the same way as when deriving the classical squeeze film flow equation (smooth surfaces), one obtains :

$$
-\frac{\mathrm{h}_{0}^{2}}{\mathrm{~h}^{3}} \frac{\mathrm{dh}}{\mathrm{dt}}=\frac{8 \mathrm{~Pa}}{3 \mu \mathrm{R}^{2}} \mathrm{~h}_{0}^{2}\left(1-2 \frac{\mathrm{a}^{2}}{\varepsilon^{2}} \frac{\mathrm{K}_{2}\left(\frac{\pi \mathrm{a}}{2 \mathrm{~h}}\right)}{\mathrm{K}_{0}\left(\frac{\pi \mathrm{a}}{2 \mathrm{~h}}\right)}+\frac{768}{\sqrt{2} \pi 5} \frac{\mathrm{hh}_{0}}{\mathrm{~h}_{0} \varepsilon} \frac{\mathrm{K}_{1}\left(\frac{\pi \varepsilon}{2 \sqrt{2} \mathrm{~h}}\right)}{\mathrm{K}_{0}\left(\frac{\pi \mathrm{a}}{2 \mathrm{~h}}\right)}\right)
$$

where $\mathrm{K}_{0}, \mathrm{~K}_{1}$ and $\mathrm{K}_{2}$ represents the modified Bessel's functions of order 0 , 1 and 2 respectively and $2 h_{0}$ stands for the initial distance between the two planes. The latter equation is an extension of the classical squeeze film equation for "rough" surfaces and reduces to the classical one (Stefan's) for the case of smooth surfaces.

The coupled problem as defined by equation (1) and the latter equation (2) (where $h_{0}$ is now written $e_{f}$ ) associated with adequate initial conditions is solved by using a Rung-Kutta method of the fourth order. The method and the associated discussion were presented in [2]. The initial value of the air layer thickness close to the slit (i.e. where sucking starts) will be taken equal to the threshold value as defined in the surface topography description. 


\section{V - DISCUSSION}

By introducing the measured roughness parameters into the mathematical model, we search for the threshold value which leads to the best agreement between the experiments and the theoretical predictions.

For that purpose, we assimilate the measured spacing between the peaks formed by the agglomerates to the distance between the cylinders which simulate roughness $(\varepsilon)$, and the peaks width to the diameter of these cylinders (2a).

Figures $7 \mathrm{a}$ and $7 \mathrm{~b}$ show the time evolution of the front radius, for two PET films of the same thickness. The "stars" correspond to the experimental values.

The experimental data are compared with the theoretical predictions for several values of the threshold, and for two film samples. The results can be summarized as follows :

1) By adjusting one single parameter, "the threshold value", a very good agreement between the experimental data and the theoretical predictions can be obtained. This is not true anymore when approaching the end of the squeeze flow process. This discrepancy may be due to some percolation phenomenon.

2) Only the highest picks (a few percent whose size is over the threshold value) seem to be important for the macroscopic flow. This tendency is true for each sample.

3) The smoother the film, the more important the highest peaks are in terms of air leakage : $7.3 \%$ of the peaks higher than 0.75 micrometers for the "rough" film and $3.3 \%$ of the peaks higher than 0.80 micrometers for the "smooth" film.

\section{VI - CONCLUSION}

The stress generated within a roll of a flexible medium such as PET film is strongly dependent on the air interlayers. The film roughness plays an important role on the air flows phenomena induced by the winding process. In order to quantify this role, an original experiment was first carried out : it consists in studying the flow of an air layer squeezed between a smooth rigid circular plate and a rough flexible PET film sample. Two parameters were introduced : (1) the thickness reduction of the air layer at the center of the disc which is representative of the film roughness for two samples of the same thickness and allows some "dynamic roughness coefficient" to be defined ; (2) the propagation of the front (i.e. the time evolution of the front radius). In this paper, a simple model, based on periodic homogeneization techniques, allows the front propagation to be predicted, assuming that the "dynamic roughness coefficient" is known and that a pertinent description of the static roughness is given. The main results is that the 
key role in a squeeze flow is played by the highest peaks. This trend is all the more marked as the film is "smooth".

The future developments would consist in giving a way to generate only a few "high enough" peaks so that to guarantee good mastering of the air interlayers without creating possible defects due to the film roughness.

\section{REFERENCES}

1. Bourgin P. and Bouquerel F.,"Winding flexible media: a global approach," Adv. Info. Storage Sys., ASME, Vol. 5, 1993, pp. 493-512.

2. Boutaous M., Bourgin P. and Woehl P.,"Periodic homogeneization techniques as a way to account for surface roughness in a confined air flow," Proc. ASME, I MECE, New York (USA), November 2001.

3. Boutaous M. and Bourgin P., "Experimental study of squeeze film between one smooth surface and one rough surface," Proceeding of the 4th Int. Conference on Web Handling, 1997, pp. 224-234, Oklahoma, USA.

4. Boutaous M.,"Influence de l'état de surface des matériaux flexibles sur leur comportement mécanique," $\mathrm{PhD}$ Thesis, Louis Pasteur University, Strasbourg, France, 1998,

5. Bayada G. and Chambat M.,"New models in the theory of the hydrodynamic lubrication of rough surfaces," Trans. ASME, J. of Lubrication Technology, Vol. 110, 1988, pp. 402-407.

6. Lee J.S. and Fung Y.C.,"Stokes flow around a cylindrical post confined between two parallel plates," Journal of Fluid Mechanics, Vol. 37, 1969, pp. 657-670.

\section{ACKNOWLEDGEMENTS}

The authors are grateful to C. Gauthier and R. Shirrer (Institut Charles Sadron, CNRS, Strasbourg) for Roughness measurements. 


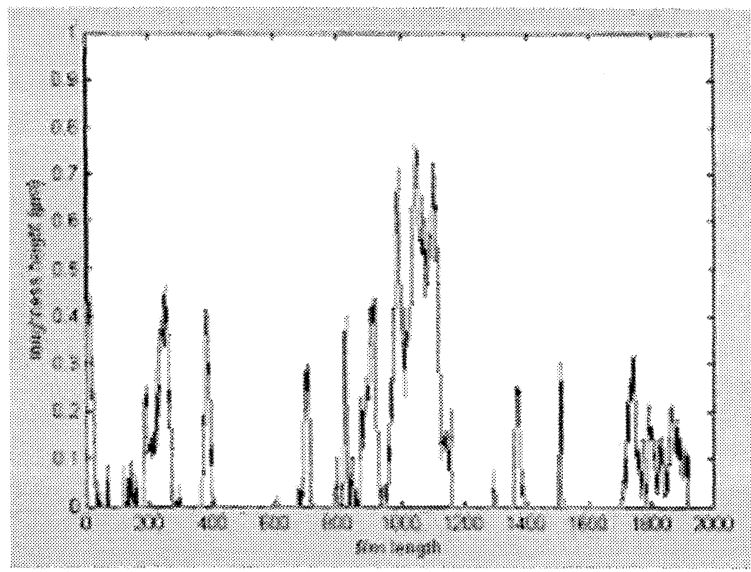

Figure 1: Typical roughness profile

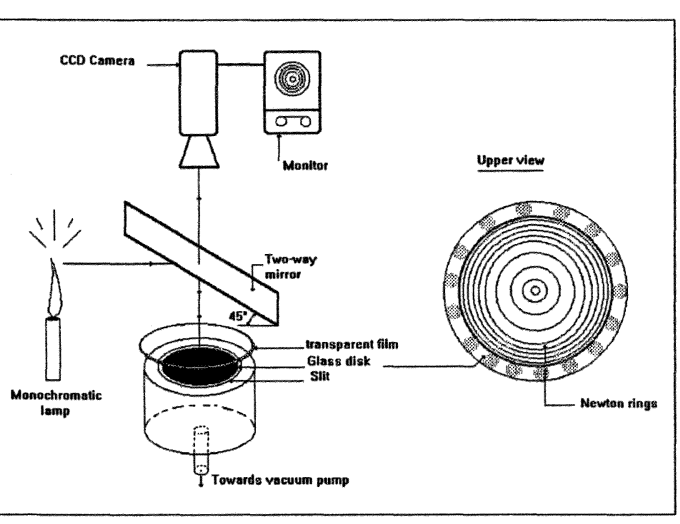

Figure 2: Squeeze flow experimental set

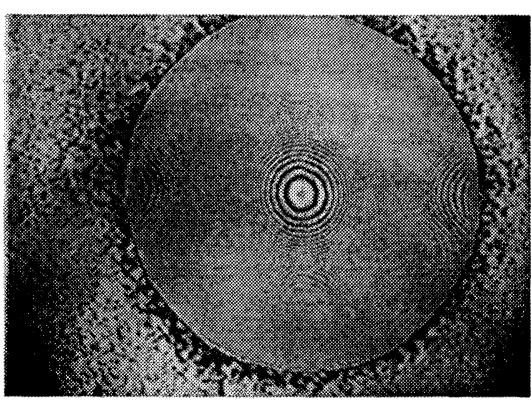

Figure 3: Evolution of the front radius and Newton rings

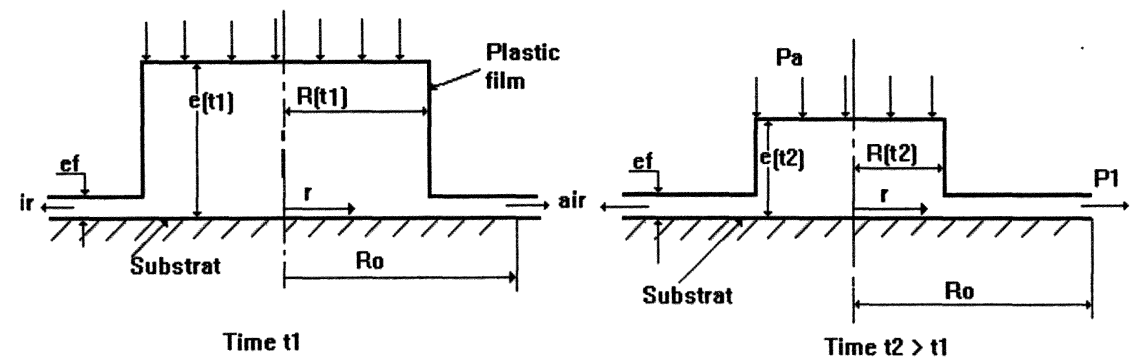

Figure 4 : Model features 


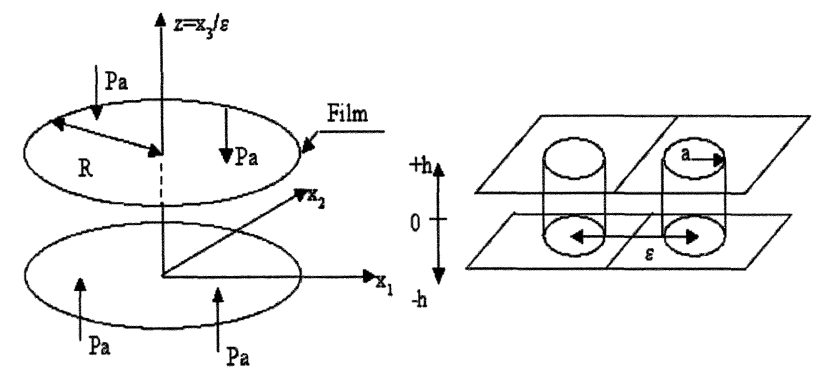

Figure 5 : Flow geometry

Figure 6: Roughness modeling

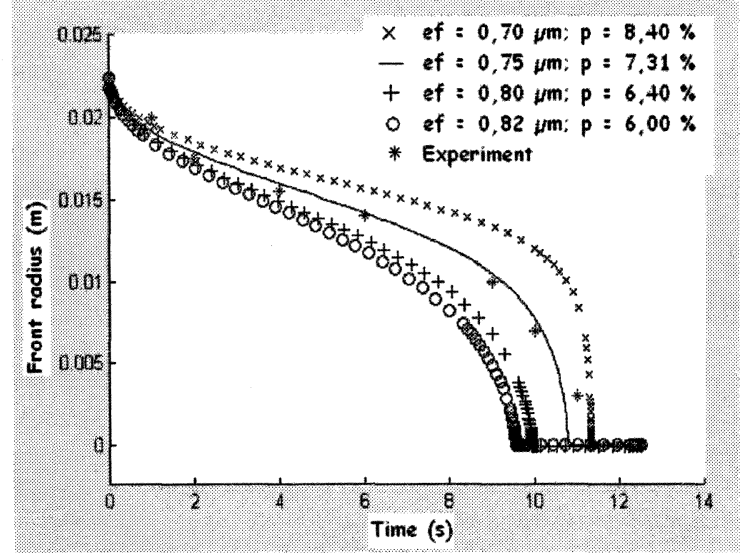

Figure 7a: Rough film: front evolution versus time

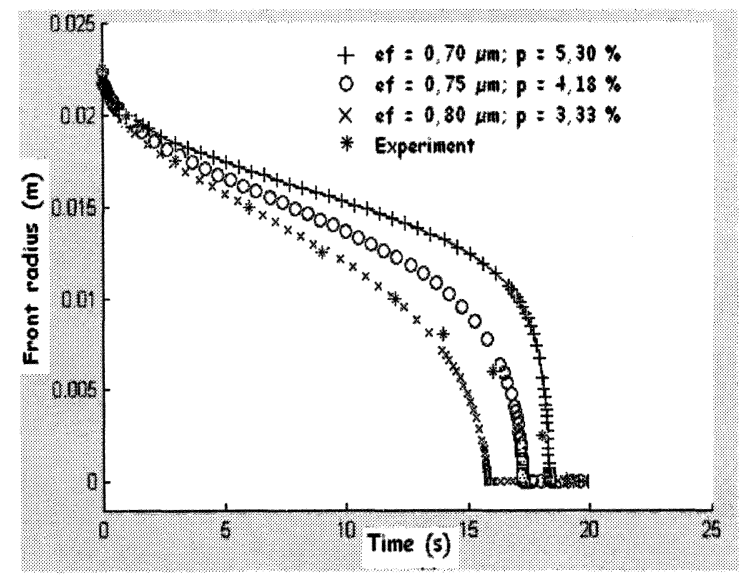

Figure 7b : Smooth film: front evolution versus

time for several threshold values. 